\title{
Stability analysis of Gauss-Seidel iterations in a partitioned simulation of fluid-structure interaction
}

\author{
Joris Degroote ${ }^{\mathrm{a}, *}$, Sebastiaan Annerel ${ }^{\mathrm{a}}$, Jan Vierendeels ${ }^{\mathrm{a}, *}$ \\ ${ }^{a}$ Department of Flow, Heat and Combustion Mechanics, Ghent University, \\ Sint-Pietersnieuwstraat 41, B-9000 Ghent, Belgium
}

\begin{abstract}
A stability analysis of Gauss-Seidel coupling iterations for partitioned simulation of fluid-structure interaction is performed for the flow in a flexible tube. In a previous study the inertia of the structure and the interaction between the segments of the structure were not taken into account. It is now demonstrated that especially the structural inertia has a significant effect on the stability of Gauss-Seidel iterations for a certain range of the time step's size.
\end{abstract}

Key words: fluid-structure interaction, partitioned, Gauss-Seidel, IQN-ILS, stability, time step

\section{Introduction}

The interaction of a fluid with a flexible structure can be simulated in either a monolithic or a partitioned way. The monolithic approach is to solve the governing equations of the flow and the structural displacement simultaneously whereas in a partitioned simulation the flow and the structural deformation are calculated with two separate solvers. Generally, a Dirichlet-Neumann decomposition of the coupled problem is employed which means that the position is imposed as a boundary condition on the fluid side of the fluid-structure interface and that the stress is imposed on the solid side.

\footnotetext{
*Corresponding author

Email addresses: Joris. Degroote@UGent . be (Joris Degroote), Sebastiaan. Annerel@UGent . be (Sebastiaan Annerel), Jan.Vierendeels@uGent. be (Jan Vierendeels)

URL: http: / /www.FSI. UGent.be/ (Joris Degroote)
} 
For a partitioned simulation of strong interaction between the fluid and the solid, e.g. blood flow in a flexible artery, a coupling algorithm is required to find the position of the interface for which the stresses on both sides of the interface are in equilibrium. One can use Gauss-Seidel iterations between the flow solver and the structural solver as coupling algorithm but these iterations converge slowly, if at all. A detailed explanation of this coupling algorithm follows later. The convergence of Gauss-Seidel iterations is improved by Aitken relaxation [1] which uses a dynamically-adapted relaxation factor. Faster convergence is obtained with Newton methods [2] or in case of black-box solvers with the Interface Generalized Minimum Residual method [3] or with quasi-Newton methods like the interface block quasi-Newton method with approximate Jacobians from least-squares models (IBQN-LS) [4] and the interface quasi-Newton method with inverse Jacobian from a least-squares model (IQN-ILS) [5]. In case of weak interaction between the fluid and the solid, e.g. aeroelasticity, an algorithm without coupling iterations between the solvers [6] can be used which means that the equilibrium on the fluid-structure interface is not enforced strictly.

The convergence of the coupling iterations depends on several parameters, such as the geometry, the time step, the structural stiffness and the ratio of the fluid density to the solid density. Förster et al. [7] analyzed the effect of these parameters on algorithms without coupling iterations. Causin et al. [8] studied algorithms with and without coupling iterations and derived the maximal relaxation factor that leads to convergence of coupling iterations as a function of the aforementioned parameters for a simplified model of blood flow in an artery and then validated the formulas with numerical experiments. Degroote et al. [9] analyzed a more simplified model for the artery and performed a modal decomposition of the interface's displacement during the coupling iterations. This Fourier error analysis demonstrates that especially error modes with a low wave number have a high amplification factor. The modal decomposition gives additional information compared to a single relaxation factor and explains why the quasi-Newton techniques with Jacobians from a least-squares model like IBQN-LS and IQN-ILS perform well.

However, the structural model in [9] neglects the mass of the structure and it is a so-called independent rings model [10] because the interaction between the segments of the tube is not taken into account. In this work, the model of the tube's wall is improved such that the structural mass and the interaction between the segments is included which leads to important new insights, among others with regard to the effect of the time step. The analytical findings from the linearized equations are confirmed by means of simulations with the original nonlinear equations. 
It has been shown in several studies $[8,7,9]$ that the instability of the coupling iterations within the time step has a physical cause. Consequently, the time discretization schemes are not expected to have much influence on the stability of the coupling iterations although they will influence the final result of the coupling iterations. To confirm this expectation, the stability analysis is performed with the backward Euler scheme for the flow equations and the Newmark scheme [11] for the structural equations but also with the Bathe composite scheme [12, 13, 14] for both the flow equations and the structural equations.

\section{Model}

\subsection{Analytical description}

The flow in an artery is simplified to the unsteady incompressible flow in a straight, flexible tube with a circular cross section and length $L$. This problem is analyzed analytically with a one-dimensional model in which gravity and viscosity are not taken into account. The flow is governed by the continuity and momentum equation, given by

$$
\begin{gathered}
\frac{\partial a}{\partial t}+\frac{\partial a u}{\partial x}=0 \\
\frac{\partial a u}{\partial t}+\frac{\partial a u^{2}}{\partial x}+\frac{1}{\rho_{f}}\left(\frac{\partial a p^{\prime}}{\partial x}-p^{\prime} \frac{\partial a}{\partial x}\right)=0
\end{gathered}
$$

with $x$ the coordinate along the axis of the tube, $a=\pi r^{2}$ the cross sectional area of the tube and $r$ the inner radius. $t$ is the time, $u$ the velocity along the axis of the tube, $p^{\prime}$ the pressure and $\rho_{f}$ the density of the fluid. The kinematic pressure $p=p^{\prime} / \rho_{f}$ is referred to as the pressure in the remainder of this text.

The structural deformation in the radial direction is determined by

$$
\rho_{s} h \frac{\partial^{2} r}{\partial t^{2}}+A \frac{\partial^{4} r}{\partial x^{4}}-B \frac{\partial^{2} r}{\partial x^{2}}+C\left(r-r_{o}\right)=\rho_{f}\left(p-p_{o}\right)
$$

with $\rho_{s}$ the solid density and $h$ the thickness of the tube's wall [10]. Axial deformations of the structure are neglected. The parameters $A$ and $B$ respectively account for the inner action of the bending in the tissue and for the tension and they depend on the properties of the structure. The parameter $C$ is equal to $\frac{E h}{r_{o}^{2}\left(1-\nu^{2}\right)}$ with $E$ the Young modulus and $\nu$ the Poisson coefficient. $r_{o}$ is the radius that corresponds with $p_{o}$.

Boundary conditions are not discussed here as they are not taken into account in a Fourier error analysis. 


\subsection{Gauss-Seidel coupling algorithm}

As in [9], Gauss-Seidel iterations between the flow problem and the structural problem are analyzed. This scheme to find the coupled solution of the time step is described below, with a superscript $k$ or $k+1$ to indicate the coupling iteration within the time step.

1. Solve Eq. (1) for the new velocity $u^{k+1}(x)$ and pressure $p^{k+1}(x)$ with a given geometry $r^{k}(x)$.

2. Solve Eq. (2) for the new geometry $r^{k+1}(x)$ with the pressure $p^{k+1}(x)$ from the preceding step.

3. Increase $k$ and return to step 1 if the iterations have not yet converged.

\subsection{Spatial discretization}

The tube is discretized in $N$ cells of length $\Delta x$ as depicted in Fig. 1. Central discretization is used for all terms in the flow equations (Eq. (1)), except for the convective term in the momentum equation which is discretized with a first-order upwind scheme. The conservation of mass and momentum in the control volume around cell center $i$ (with $i=1, \ldots, N$ ) is given by

$$
\begin{gathered}
\Delta x \frac{\partial a_{i}}{\partial t}+u_{i+1 / 2} a_{i+1 / 2}-u_{i-1 / 2} a_{i-1 / 2}-\alpha\left(p_{i+1}-2 p_{i}+p_{i-1}\right)=0 \\
\Delta x \frac{\partial u_{i} a_{i}}{\partial t}+u_{i} u_{i+1 / 2} a_{i+1 / 2}-u_{i-1} u_{i-1 / 2} a_{i-1 / 2} \\
+\frac{1}{2}\left(a_{i+1 / 2}\left(p_{i+1}-p_{i}\right)+a_{i-1 / 2}\left(p_{i}-p_{i-1}\right)\right)=0
\end{gathered}
$$

for $u_{i} \geq 0$. The subscripts $i \pm 1 / 2$ denote the value at the cell interfaces, $u_{i-1 / 2}=\left(u_{i-1}+u_{i}\right) / 2$ and $u_{i+1 / 2}=\left(u_{i}+u_{i+1}\right) / 2$. The pressure stabilization term with coefficient $\alpha=a_{o} /\left(u_{o}+\Delta x / \Delta t\right)$ is added in the continuity equation to inhibit pressure wiggles due to the central discretization of the pressure in the momentum equation, with $u_{o}$ the reference flow velocity. This stabilization term can be written as

$$
\frac{a_{o}}{u_{o}+\Delta x / \Delta t}\left(\left.\Delta x^{2} \frac{\partial^{2} p}{\partial x^{2}}\right|_{i}+O\left(\Delta x^{4}\right)\right)
$$

on Cartesian grids. For large $\Delta t$, the stabilization term scales with $O(\Delta x)$ with respect to the other terms in Eq. (3a) and for small $\Delta t$, the stabilization term scales 
with $O(\Delta t)$ with respect to the other terms. Thus, the stabilization term does not affect the accuracy of the scheme because the other terms are also first-order accurate. In [15], the suppression of pressure wiggles by this term is investigated with Fourier analysis and its implementation with higher-order accuracy on non-Cartesian grids using a finite volume discretization is described. In the finite element context, the inf-sup condition can be used to ensure the stability and optimality of a discretization scheme [16].

Eq. (2) for the structure is discretized in space with the central difference method, giving

$$
\begin{aligned}
\rho_{s} h \frac{\partial^{2} r_{i}}{\partial t^{2}}+\frac{A}{\Delta x^{4}} & \left(r_{i+2}-4 r_{i+1}+6 r_{i}-4 r_{i-1}+r_{i-2}\right) \\
& -\frac{B}{\Delta x^{2}}\left(r_{i+1}-2 r_{i}+r_{i-1}\right)+C\left(r_{i}-r_{o}\right)=\rho_{f}\left(p_{i}-p_{o}\right) .
\end{aligned}
$$

\subsection{Time discretization with the backward Euler and Newmark scheme}

The spatially discrete equations are subsequently discretized in time. In this section, the backward Euler scheme is used for the time discretization of the flow equations. The first superscript $n$ denotes the time step and the second superscript $k$ indicates the coupling iteration within time step $n+1$. The time discretization of Eq. (3) is thus given by

$$
\begin{aligned}
& \frac{\Delta x}{\Delta t}\left(a_{i}^{n+1, k}-a_{i}^{n}\right)+u_{i+1 / 2}^{n+1, k+1} a_{i+1 / 2}^{n+1, k}-u_{i-1 / 2}^{n+1, k+1} a_{i-1 / 2}^{n+1, k} \\
& -\alpha\left(p_{i+1}^{n+1, k+1}-2 p_{i}^{n+1, k+1}+p_{i-1}^{n+1, k+1}\right)=0 \quad \text { (6a) } \\
& \frac{\Delta x}{\Delta t}\left(u_{i}^{n+1, k+1} a_{i}^{n+1, k}-u_{i}^{n} a_{i}^{n}\right)+u_{i}^{n+1, k+1} u_{i+1 / 2}^{n+1, k+1} a_{i+1 / 2}^{n+1, k}-u_{i-1}^{n+1, k+1} u_{i-1 / 2}^{n+1, k+1} a_{i-1 / 2}^{n+1, k} \\
& +\frac{1}{2}\left(a_{i+1 / 2}^{n+1, k}\left(p_{i+1}^{n+1, k+1}-p_{i}^{n+1, k+1}\right)+a_{i-1 / 2}^{n+1, k}\left(p_{i}^{n+1, k+1}-p_{i-1}^{n+1, k+1}\right)\right)=0 \quad \text { (6b) }
\end{aligned}
$$

for $u_{i} \geq 0$ with $\Delta t$ the time step.

Eq. (5) for the structure is discretized in time with the Newmark method [11], 
giving

$$
\begin{aligned}
\frac{\rho_{s} h}{\beta \Delta t^{2}} & r_{i}^{n+1, k+1}+\frac{A}{\Delta x^{4}}\left(r_{i+2}^{n+1, k+1}-4 r_{i+1}^{n+1, k+1}+6 r_{i}^{n+1, k+1}-4 r_{i-1}^{n+1, k+1}+r_{i-2}^{n+1, k+1}\right) \\
& -\frac{B}{\Delta x^{2}}\left(r_{i+1}^{n+1, k+1}-2 r_{i}^{n+1, k+1}+r_{i-1}^{n+1, k+1}\right)+C\left(r_{i}^{n+1, k+1}-r_{o}\right) \\
= & \rho_{f}\left(p_{i}^{n+1, k+1}-p_{o}\right)+\rho_{s} h\left(\frac{1}{\beta \Delta t^{2}} r_{i}^{n}+\frac{1}{\beta \Delta t} \dot{r}_{i}^{n}+\left(\frac{1}{2 \beta}-1\right) \ddot{r}_{i}^{n}\right)
\end{aligned}
$$

in which an over-dot signifies a time derivative. Once the coupling iterations within time step $n+1$ have converged, the corresponding acceleration and velocity are calculated as

$$
\begin{aligned}
\ddot{r}_{i}^{n+1} & =\frac{1}{\beta \Delta t^{2}}\left(r_{i}^{n+1}-r_{i}^{n}\right)-\frac{1}{\beta \Delta t} \dot{r}_{i}^{n}-\left(\frac{1}{2 \beta}-1\right) \ddot{r}_{i}^{n} \\
\dot{r}_{i}^{n+1} & =\dot{r}_{i}^{n}+\Delta t(1-\gamma) \ddot{r}_{i}^{n}+\Delta t \gamma \ddot{r}_{i}^{n+1} .
\end{aligned}
$$

The Newmark parameters $\beta$ and $\gamma$ are chosen such that $\gamma \geq \frac{1}{2}$ and $\beta \geq \frac{1}{4}\left(\frac{1}{2}+\gamma\right)^{2}$ which results in an unconditionally stable integration scheme.

\subsection{Time discretization with the composite scheme}

The time discretization schemes employed in the previous section are incompatible because identical displacements on the fluid and solid side of the interface do not result in identical velocities and accelerations on both sides of the interface. To verify that the outcome of the stability analysis is not influenced significantly by this incompatibility, the flow equations and the structural equations are also discretized in time with a compatible time discretization scheme, namely the composite scheme $[12,13,14]$. The composite scheme divides the time step $\Delta t$ in two sub-steps by means of an intermediate point at $\delta \Delta t(0<\delta<1)$ and uses the trapezoidal rule in the first sub-step and the 3-point backward Euler scheme in the second sub-step.

For the composite scheme, values at the beginning of the time step are indicated with a superscript $n$, values at the intermediate point with a superscript $n+\delta$ and values at the end of the time step with a superscript $n+1$. Because the time step is divided in two sub-steps, the Gauss-Seidel coupling algorithm has to be applied twice and coupling iterations have to be performed in the first sub-step (superscript $k$ ) and in the second sub-step (superscript $k^{\prime}$ ). The stability of the coupling iterations in both sub-steps will be analyzed. 
The first sub-step of the composite scheme for the time discretization of Eq. (3) is given by

$$
\begin{aligned}
& \frac{\Delta x}{\delta \Delta t}\left(a_{i}^{n+\delta, k}-a_{i}^{n}\right)+u_{i+1 / 2}^{n+\delta / 2, k+1} a_{i+1 / 2}^{n+\delta / 2, k}-u_{i-1 / 2}^{n+\delta / 2, k+1} a_{i-1 / 2}^{n+\delta / 2, k} \\
& \left.-\alpha\left(p_{i+1}^{n+\delta / 2, k+1}-2 p_{i}^{n+\delta / 2, k+1}+p_{i-1}^{n+\delta / 2, k+1}\right)=0 \quad \text { (8a }\right) \\
& \frac{\Delta x}{\delta \Delta t}\left(u_{i}^{n+\delta, k+1} a_{i}^{n+\delta, k}-u_{i}^{n} a_{i}^{n}\right)+u_{i}^{n+\delta / 2, k+1} u_{i+1 / 2}^{n+\delta / 2, k+1} a_{i+1 / 2}^{n+\delta / 2, k}-u_{i-1}^{n+\delta / 2, k+1} u_{i-1 / 2}^{n+\delta / 2, k+1} a_{i-1 / 2}^{n+\delta / 2, k} \\
& +\frac{1}{2}\left(a_{i+1 / 2}^{n+\delta / 2, k}\left(p_{i+1}^{n+\delta / 2, k+1}-p_{i}^{n+\delta / 2, k+1}\right)+a_{i-1 / 2}^{n+\delta / 2, k}\left(p_{i}^{n+\delta / 2, k+1}-p_{i-1}^{n+\delta / 2, k+1}\right)\right)=0
\end{aligned}
$$

for $u_{i} \geq 0$ with $u^{n+\delta / 2, k+1}=\left(u^{n}+u^{n+\delta, k+1}\right) / 2$ and analogously for $a^{n+\delta / 2, k}$ and $p^{n+\delta / 2, k+1}$.

For the structure, the first sub-step in the composite time discretization of Eq. (5) results in

$$
\begin{gathered}
\frac{4 \rho_{s} h}{\delta^{2} \Delta t^{2}} r_{i}^{n+\delta, k+1}+\frac{A}{\Delta x^{4}}\left(r_{i+2}^{n+\delta, k+1}-4 r_{i+1}^{n+\delta, k+1}+6 r_{i}^{n+\delta, k+1}-4 r_{i-1}^{n+\delta, k+1}+r_{i-2}^{n+\delta, k+1}\right) \\
-\frac{B}{\Delta x^{2}}\left(r_{i+1}^{n+\delta, k+1}-2 r_{i}^{n+\delta, k+1}+r_{i-1}^{n+\delta, k+1}\right)+C\left(r_{i}^{n+\delta, k+1}-r_{o}\right) \\
=\rho_{f}\left(p_{i}^{n+\delta, k+1}-p_{o}\right)+\rho_{s} h\left(\frac{4}{\delta^{2} \Delta t^{2}} r_{i}^{n}+\frac{4}{\delta \Delta t} \dot{r}_{i}^{n}+\ddot{r}_{i}^{n}\right)
\end{gathered}
$$

in which an over-dot again signifies a time derivative. Gauss-Seidel coupling iterations between Eq. (8) and Eq. (9a) are performed and once these coupling iterations within the first sub-step have converged, the corresponding velocity and acceleration are calculated as

$$
\begin{aligned}
\dot{r}_{i}^{n+\delta} & =\frac{2}{\delta \Delta t}\left(r_{i}^{n+\delta}-r_{i}^{n}\right)-\dot{r}_{i}^{n} . \\
\ddot{r}_{i}^{n+\delta} & =\frac{4}{\delta^{2} \Delta t^{2}}\left(r_{i}^{n+\delta}-r_{i}^{n}\right)-\frac{4}{\delta \Delta t} \dot{r}_{i}^{n}-\ddot{r}_{i}^{n}
\end{aligned}
$$

In the second sub-step, all values from the previous time step (superscript $n$ ) and the end of the first sub-step (superscript $n+\delta$ ) are known. The flow equations 
(Eq. (3)) are discretized in time as

$$
\begin{gathered}
\frac{\Delta x}{\left(1-\eta_{1}\right) \Delta t}\left(a_{i}^{n+1, k^{\prime}}-\left(1-\eta_{2}\right) a_{i}^{n}-\eta_{2} a_{i}^{n+\delta}\right)+u_{i+1 / 2}^{n+1, k^{\prime}+1} a_{i+1 / 2}^{n+1, k^{\prime}}-u_{i-1 / 2}^{n+1, k^{\prime}+1} a_{i-1 / 2}^{n+1, k^{\prime}} \\
-\alpha\left(p_{i+1}^{n+1, k^{\prime}+1}-2 p_{i}^{n+1, k^{\prime}+1}+p_{i-1}^{n+1, k^{\prime}+1}\right)=0 \quad \text { (10a) } \\
\frac{\Delta x}{\left(1-\eta_{1}\right) \Delta t}\left(u_{i}^{n+1, k^{\prime}+1} a_{i}^{n+1, k^{\prime}}-\left(1-\eta_{2}\right) u_{i}^{n} a_{i}^{n}-\eta_{2} u_{i}^{n+\delta} a_{i}^{n+\delta}\right) \\
\quad+u_{i}^{n+1, k^{\prime}+1} u_{i+1 / 2}^{n+1, k^{\prime}+1} a_{i+1 / 2}^{n+1, k^{\prime}}-u_{i-1}^{n+1, k^{\prime}+1} u_{i-1 / 2}^{n+1, k^{\prime}+1} a_{i-1 / 2}^{n+1, k^{\prime}} \\
+\frac{1}{2}\left(a_{i+1 / 2}^{n+1, k^{\prime}}\left(p_{i+1}^{n+1, k^{\prime}+1}-p_{i}^{n+1, k^{\prime}+1}\right)+a_{i-1 / 2}^{n+1, k^{\prime}}\left(p_{i}^{n+1, k^{\prime}+1}-p_{i-1}^{n+1, k^{\prime}+1}\right)\right)=0
\end{gathered}
$$

for $u_{i} \geq 0$. The parameters $\eta$ in the previous equations are calculated as

$$
\begin{aligned}
\eta_{1} & =\frac{1}{2-\delta} \\
\eta_{2} & =\frac{\eta_{1}^{2}}{2 \eta_{1}-1} .
\end{aligned}
$$

The time discretization of the structural equations (Eq. (5)) in the second substep of the composite scheme is given by

$$
\begin{aligned}
& \rho_{s} h \lambda_{3}^{2} r_{i}^{n+1, k^{\prime}+1}+\frac{A}{\Delta x^{4}}\left(r_{i+2}^{n+1, k^{\prime}+1}-4 r_{i+1}^{n+1, k^{\prime}+1}+6 r_{i}^{n+1, k^{\prime}+1}-4 r_{i-1}^{n+1, k^{\prime}+1}+r_{i-2}^{n+1, k^{\prime}+1}\right) \\
& \quad-\frac{B}{\Delta x^{2}}\left(r_{i+1}^{n+1, k^{\prime}+1}-2 r_{i}^{n+1, k^{\prime}+1}+r_{i-1}^{n+1, k^{\prime}+1}\right)+C\left(r_{i}^{n+1, k^{\prime}+1}-r_{o}\right) \\
& =\rho_{f}\left(p_{i}^{n+1, k^{\prime}+1}-p_{o}\right)+\rho_{s} h\left(-\lambda_{1} \dot{r}_{i}^{n}-\lambda_{2} \dot{r}_{i}^{n+\delta}-\lambda_{3} \lambda_{1} r_{i}^{n}-\lambda_{3} \lambda_{2} r_{i}^{n+\delta}\right) . \quad \text { (12a) }
\end{aligned}
$$

Gauss-Seidel coupling iterations are also performed between Eq. (10) and Eq. (12a). When the coupling iterations within the second sub-step have converged, the corresponding velocity and acceleration are calculated as

$$
\begin{aligned}
& \dot{r}_{i}^{n+1}=\lambda_{1} r_{i}^{n}+\lambda_{2} r_{i}^{n+\delta}+\lambda_{3} r_{i}^{n+1} \\
& \ddot{r}_{i}^{n+1}=\lambda_{1} \dot{r}_{i}^{n}+\lambda_{2} \dot{r}_{i}^{n+\delta}+\lambda_{3} \dot{r}_{i}^{n+1} .
\end{aligned}
$$


The parameters $\lambda$ used in the second sub-step of the composite time discretization of the structural equations are defined as

$$
\begin{aligned}
& \lambda_{1}=\frac{1-\delta}{\delta \Delta t} \\
& \lambda_{2}=\frac{-1}{(1-\delta) \delta \Delta t} \\
& \lambda_{3}=\frac{2-\delta}{(1-\delta) \Delta t} .
\end{aligned}
$$

\section{Linear error analysis}

\subsection{Time discretization with the backward Euler and Newmark scheme}

The stability of the Gauss-Seidel coupling algorithm is now determined with Fourier error analysis on the flow equations with backward Euler time discretization and on the structural equations with Newmark time discretization. Therefore, the velocity, pressure and inner radius of the tube in Eq. (6) and Eq. (7) are substituted by the sum of the coupled solution and the remaining error in the coupling iteration (indicated with a tilde). The coupled solution is in turn linearized as the sum of the reference value (subscript $o$ ) and a perturbation (indicated with a hat). For the velocity, this gives

$$
u_{i}^{k}=u_{o}+\hat{u}_{i}+\tilde{u}_{i}^{k}
$$

and analogously for the pressure and radius.

Subsequently, $a$ is replaced by $\pi r^{2}$ and all equations are linearized by neglecting the non-linear combinations of the error terms and the perturbations. Because the equations linearized around $u_{o}, p_{o}$ and $r_{o}$ are satisfied by the coupled solution, all perturbations and the values from the previous time step cancel out which results in the following equations for the error terms

$$
\begin{aligned}
& \begin{aligned}
\frac{\Delta x}{\Delta t} 2 r_{o} \tilde{r}_{i}^{k}+2 u_{o} r_{o}\left(\tilde{r}_{i+1 / 2}^{k}-\tilde{r}_{i-1 / 2}^{k}\right)+ & r_{o}^{2}\left(\tilde{u}_{i+1 / 2}^{k+1}-\tilde{u}_{i-1 / 2}^{k+1}\right) \\
& -\alpha^{\prime}\left(\tilde{p}_{i+1}^{k+1}-2 \tilde{p}_{i}^{k+1}+\tilde{p}_{i-1}^{k+1}\right)=0
\end{aligned} \\
& \begin{aligned}
\frac{\Delta x}{\Delta t}\left(2 u_{o} r_{o} \tilde{r}_{i}^{k}+r_{o}^{2} \tilde{u}_{i}^{k+1}\right)+2 u_{o}^{2} r_{o}\left(\tilde{r}_{i+1 / 2}^{k}-\tilde{r}_{i-1 / 2}^{k}\right) \\
\quad+u_{o} r_{o}^{2}\left(\tilde{u}_{i+1 / 2}^{k+1}+\tilde{u}_{i}^{k+1}-\tilde{u}_{i-1 / 2}^{k+1}-\tilde{u}_{i-1}^{k+1}\right)+\frac{r_{o}^{2}}{2}\left(\tilde{p}_{i+1}^{k+1}-\tilde{p}_{i-1}^{k+1}\right)=0
\end{aligned}
\end{aligned}
$$




$$
\begin{aligned}
\frac{\rho_{s} h}{\beta \Delta t^{2}} \tilde{r}_{i}^{k+1}+\frac{A}{\Delta x^{4}}\left(\tilde{r}_{i+2}^{k+1}-4 \tilde{r}_{i+1}^{k+1}+6 \tilde{r}_{i}^{k+1}-4 \tilde{r}_{i-1}^{k+1}+\tilde{r}_{i-2}^{k+1}\right) & \\
& -\frac{B}{\Delta x^{2}}\left(\tilde{r}_{i+1}^{k+1}-2 \tilde{r}_{i}^{k+1}+\tilde{r}_{i-1}^{k+1}\right)+C \tilde{r}_{i}^{k+1}=\rho_{f} \tilde{p}_{i}^{k+1}
\end{aligned}
$$

with $\alpha^{\prime}=\alpha / \pi$. All variables in the previous equations are at time level $n+1$ and therefore the first superscript has been omitted. Eq. (7b) and Eq. (7c) are only used at the end of the time step and hence they are of no importance for the stability of the coupling iterations within the time step, so that $\gamma$ is not a parameter therein.

The error terms are expanded as the sum of $N$ Fourier modes, resulting in

$$
\tilde{u}_{i}^{k}=\frac{1}{N} \sum_{\ell=0}^{N-1} \bar{u}_{\ell}^{k} \exp \left(\jmath \omega_{\ell} i \Delta x\right)
$$

for the error on the velocity with $\jmath=\sqrt{-1}$ and $\omega_{\ell}=2 \pi \ell / L$ the angular wave number. The amplification of every wave number can be studied separately as Eq. (15) is linear in $\tilde{u}, \tilde{r}$ and $\tilde{p}$. Therefore, $\tilde{u}_{i}^{k}$ is substituted by $\bar{u}_{\ell}^{k} \exp \left(\jmath \omega_{\ell} i \Delta x\right)$ and analogously for the error on the pressure and the radius. The product $\omega_{\ell} \Delta x$ is further denoted as $\vartheta_{\ell}$ and for clarity the bar and the subscript $\ell$ are omitted.

$$
\begin{aligned}
& \frac{\Delta x}{\Delta t} 2 r_{o} r^{k}+2 u_{o} r_{o} \jmath \sin (\vartheta) r^{k}+r_{o}^{2} \jmath \sin (\vartheta) u^{k+1}-2 \alpha^{\prime}(\cos (\vartheta)-1) p^{k+1}=0 \\
& \begin{aligned}
& \frac{\Delta x}{\Delta t}\left(2 u_{o} r_{o} r^{k}+r_{o}^{2} u^{k+1}\right)+2 u_{o}^{2} r_{o} \jmath \sin (\vartheta) r^{k} \\
& \quad+u_{o} r_{o}^{2}(1+\jmath \sin (\vartheta)-\exp (-\jmath \vartheta)) u^{k+1}+r_{o}^{2} \jmath \sin (\vartheta) p^{k+1}=0 \\
&\left(\frac{\rho_{s} h}{\beta \Delta t^{2}}+\frac{4 A}{\Delta x^{4}}(\cos (\vartheta)-1)^{2}-\frac{2 B}{\Delta x^{2}}(\cos (\vartheta)-1)+C\right) r^{k+1}=\rho_{f} p^{k+1}
\end{aligned}
\end{aligned}
$$

By combining these equations, the amplification factor $\mu$ of every mode in the error on the radius is calculated as

$$
\left|\frac{r^{k+1}}{r^{k}}\right|=\mu=\left|\mu_{1} \mu_{2}\right|
$$

with

$$
\begin{aligned}
& \mu_{1}=\frac{\frac{\rho_{f}}{\rho_{s}}}{\frac{\rho_{f} r_{o}^{2}\left(1-\nu^{2}\right)}{E \beta \Delta t^{2}}+\frac{4 A r_{o}^{2}\left(1-\nu^{2}\right)}{E h \Delta x^{4}} \frac{\rho_{f}}{\rho_{s}}(\cos (\vartheta)-1)^{2}} \\
& -\frac{2 B r_{o}^{2}\left(1-\nu^{2}\right)}{E h \Delta x^{2}} \frac{\rho_{f}}{\rho_{s}}(\cos (\vartheta)-1)+\frac{\rho_{f}}{\rho_{s}}
\end{aligned}
$$


and

$$
\begin{aligned}
& \left(\frac{\Delta x}{\Delta t}+u_{o}(\jmath \sin (\vartheta)+1-\exp (-\jmath \vartheta))\right) \\
& \cdot\left(2 \frac{\Delta x}{\Delta t}+2 u_{o} \jmath \sin (\vartheta)\right) \\
& \mu_{2}=\frac{\rho_{f} r_{o}\left(1-\nu^{2}\right)}{E h} \cdot \frac{-\left(2 \frac{\Delta x}{\Delta t} u_{o} \jmath \sin (\vartheta)-2 u_{o}^{2} \sin ^{2}(\vartheta)\right)}{\left(\frac{\Delta x}{\Delta t}+u_{o}(\jmath \sin (\vartheta)+1-\exp (-\jmath \vartheta))\right)} \\
& \cdot\left(\frac{2}{u_{o}+\Delta x / \Delta t}(\cos (\vartheta)-1)\right)-\sin ^{2}(\vartheta)
\end{aligned}
$$

The term $\mu_{2}$ in the above equation is identical to the error amplification factor that has been derived in [9] using a simple structural model without inertia and without interaction between the segments of the tube. The structural model that is presented in this paper (Eq. (2)) with inertia and with interaction between the segments results in additional contributions to the error amplification which are all grouped in a new term $\mu_{1}$ such that the complete error amplification factor is obtained as the product of $\mu_{1}$ and $\mu_{2}$. Consequently, the results of the preceding analysis in [9] are confirmed and its conclusions remain valid. $\alpha^{\prime}$ has been substituted by

$$
\alpha^{\prime}=\frac{\left(r_{o}\right)^{2}}{u_{o}+\Delta x / \Delta t}
$$

in the previous equation because $\alpha^{\prime}$ cannot be altered independently as a parameter.

\subsection{Time discretization with the composite scheme}

The stability of the Gauss-Seidel coupling iterations between the flow equations and the structural equations in the first and second sub-step of the composite time discretization is analyzed in this section in the same way as it has been done for the backward Euler and Newmark time discretization in the previous section. The variables in Eq. (8) and Eq. (9) for the first sub-step are decomposed as the sum of a reference, a perturbation and the remaining error as described in Eq. (14) and subsequently all equations are linearized. The perturbations and the values from the previous time step cancel out such that only error terms at time level $n+\delta$ remain and consequently the first superscript can be omitted. The resulting equations for the error terms in the first sub-step are

$$
\begin{aligned}
\frac{2 \Delta x}{\delta \Delta t} 2 r_{o} \tilde{r}_{i}^{k}+2 u_{o} r_{o}\left(\tilde{r}_{i+1 / 2}^{k}-\tilde{r}_{i-1 / 2}^{k}\right)+ & r_{o}^{2}\left(\tilde{u}_{i+1 / 2}^{k+1}-\tilde{u}_{i-1 / 2}^{k+1}\right) \\
& -\alpha^{\prime}\left(\tilde{p}_{i+1}^{k+1}-2 \tilde{p}_{i}^{k+1}+\tilde{p}_{i-1}^{k+1}\right)=0
\end{aligned}
$$




$$
\begin{aligned}
& \frac{2 \Delta x}{\delta \Delta t}\left(2 u_{o} r_{o} \tilde{r}_{i}^{k}+r_{o}^{2} \tilde{u}_{i}^{k+1}\right)+2 u_{o}^{2} r_{o}\left(\tilde{r}_{i+1 / 2}^{k}-\tilde{r}_{i-1 / 2}^{k}\right) \\
& +u_{o} r_{o}^{2}\left(\tilde{u}_{i+1 / 2}^{k+1}+\tilde{u}_{i}^{k+1}-\tilde{u}_{i-1 / 2}^{k+1}-\tilde{u}_{i-1}^{k+1}\right)+\frac{r_{o}^{2}}{2}\left(\tilde{p}_{i+1}^{k+1}-\tilde{p}_{i-1}^{k+1}\right)=0 \\
& \frac{4 \rho_{s} h}{\delta^{2} \Delta t^{2}} \tilde{r}_{i}^{k+1}+\frac{A}{\Delta x^{4}}\left(\tilde{r}_{i+2}^{k+1}-4 \tilde{r}_{i+1}^{k+1}+6 \tilde{r}_{i}^{k+1}-4 \tilde{r}_{i-1}^{k+1}+\tilde{r}_{i-2}^{k+1}\right) \\
& \quad-\frac{B}{\Delta x^{2}}\left(\tilde{r}_{i+1}^{k+1}-2 \tilde{r}_{i}^{k+1}+\tilde{r}_{i-1}^{k+1}\right)+C \tilde{r}_{i}^{k+1}=\rho_{f} \tilde{p}_{i}^{k+1}
\end{aligned}
$$

with $\alpha^{\prime}=\alpha / \pi$. The error terms in the first sub-step are then expanded as the sum of $N$ Fourier modes using Eq. (16) and every one of these modes can be analyzed separately because the previous equations are linear. By combining the equations for the coefficients of the Fourier modes as in the previous section, the amplification factor $\mu$ during the coupling iterations in the first sub-step is obtained.

$$
\begin{aligned}
& \mu_{1}=\frac{\frac{\rho_{f}}{\rho_{s}}}{\frac{4 \rho_{f} r_{o}^{2}\left(1-\nu^{2}\right)}{E \delta^{2} \Delta t^{2}}+\frac{4 A r_{o}^{2}\left(1-\nu^{2}\right)}{E h \Delta x^{4}} \frac{\rho_{f}}{\rho_{s}}(\cos (\vartheta)-1)^{2}} \\
& -\frac{2 B r_{o}^{2}\left(1-\nu^{2}\right)}{E h \Delta x^{2}} \frac{\rho_{f}}{\rho_{s}}(\cos (\vartheta)-1)+\frac{\rho_{f}}{\rho_{s}}
\end{aligned}
$$

and

$$
\begin{aligned}
& \left(\frac{2 \Delta x}{\delta \Delta t}+u_{o}(\jmath \sin (\vartheta)+1-\exp (-\jmath \vartheta))\right) \\
& \text {. }\left(2 \frac{2 \Delta x}{\delta \Delta t}+2 u_{o} \jmath \sin (\vartheta)\right) \\
& \mu_{2}=\frac{\rho_{f} r_{o}\left(1-\nu^{2}\right)}{E h} \cdot \frac{-\left(2 \frac{2 \Delta x}{\delta \Delta t} u_{o} \jmath \sin (\vartheta)-2 u_{o}^{2} \sin ^{2}(\vartheta)\right)}{\left(\frac{2 \Delta x}{\delta \Delta t}+u_{o}(\jmath \sin (\vartheta)+1-\exp (-\jmath \vartheta))\right)} \\
& \cdot\left(\frac{2}{u_{o}+\Delta x / \Delta t}(\cos (\vartheta)-1)\right)-\sin ^{2}(\vartheta)
\end{aligned}
$$

For the analysis of the coupling iterations in the second sub-step, the variables in Eq. (10) and Eq. (12) are again decomposed as the sum of a reference, a perturbation and the remaining error, followed by linearization of all equations. The perturbations, the values from the previous time step (superscript $n$ ) and the values from the first sub-step (superscript $n+\delta$ ) cancel out such that only error terms at time level $n+1$ remain and consequently the first superscript can again be omitted. The resulting equations for the error terms in the second sub-step are

$$
\begin{array}{r}
\frac{\Delta x}{\left(1-\eta_{1}\right) \Delta t} 2 r_{o} \tilde{r}_{i}^{k^{\prime}}+2 u_{o} r_{o}\left(\tilde{r}_{i+1 / 2}^{k^{\prime}}-\tilde{r}_{i-1 / 2}^{k^{\prime}}\right)+r_{o}^{2}\left(\tilde{u}_{i+1 / 2}^{k^{\prime}+1}-\tilde{u}_{i-1 / 2}^{k^{\prime}+1}\right) \\
-\alpha^{\prime}\left(\tilde{p}_{i+1}^{k^{\prime}+1}-2 \tilde{p}_{i}^{k^{\prime}+1}+\tilde{p}_{i-1}^{k^{\prime}+1}\right)=0
\end{array}
$$




$$
\begin{gathered}
\frac{\Delta x}{\left(1-\eta_{1}\right) \Delta t}\left(2 u_{o} r_{o} \tilde{r}_{i}^{k^{\prime}}+r_{o}^{2} \tilde{u}_{i}^{k^{\prime}+1}\right)+2 u_{o}^{2} r_{o}\left(\tilde{r}_{i+1 / 2}^{k^{\prime}}-\tilde{r}_{i-1 / 2}^{k^{\prime}}\right) \\
+u_{o} r_{o}^{2}\left(\tilde{u}_{i+1 / 2}^{k^{\prime}+1}+\tilde{u}_{i}^{k^{\prime}+1}-\tilde{u}_{i-1 / 2}^{k^{\prime}+1}-\tilde{u}_{i-1}^{k^{\prime}+1}\right)+\frac{r_{o}^{2}}{2}\left(\tilde{p}_{i+1}^{k^{\prime}+1}-\tilde{p}_{i-1}^{k^{\prime}+1}\right)=0 \\
\rho_{s} h \lambda_{3}^{2} \tilde{r}_{i}^{k^{\prime}+1}+\frac{A}{\Delta x^{4}}\left(\tilde{r}_{i+2}^{k^{\prime}+1}-4 \tilde{r}_{i+1}^{k^{\prime}+1}+6 \tilde{r}_{i}^{k^{\prime}+1}-4 \tilde{r}_{i-1}^{k^{\prime}+1}+\tilde{r}_{i-2}^{k^{\prime}+1}\right) \\
-\frac{B}{\Delta x^{2}}\left(\tilde{r}_{i+1}^{k^{\prime}+1}-2 \tilde{r}_{i}^{k^{\prime}+1}+\tilde{r}_{i-1}^{k^{\prime}+1}\right)+C \tilde{r}_{i}^{k^{\prime}+1}=\rho_{f} \tilde{p}_{i}^{k^{\prime}+1} .
\end{gathered}
$$

From the equations above, the amplification factor $\mu$ during the coupling iterations in the second sub-step is derived after substitution of the error terms by their Fourier decomposition.

$$
\mu_{1}=\frac{\frac{\rho_{f}}{\rho_{s}}}{\frac{\lambda_{3}^{2} \rho_{f} r_{o}^{2}\left(1-\nu^{2}\right)}{E}+\frac{4 A r_{o}^{2}\left(1-\nu^{2}\right)}{E h \Delta x^{4}} \frac{\rho_{f}}{\rho_{s}}(\cos (\vartheta)-1)^{2}}
$$

and

$$
\begin{gathered}
\left(\frac{\Delta x}{\left(1-\eta_{1}\right) \Delta t}+u_{o}(\jmath \sin (\vartheta)+1-\exp (-\jmath \vartheta))\right) \\
\cdot\left(2 \frac{\Delta x}{\left(1-\eta_{1}\right) \Delta t}+2 u_{o} \jmath \sin (\vartheta)\right) \\
\mu_{2}=\frac{\rho_{f} r_{o}\left(1-\nu^{2}\right)}{E h} \cdot \frac{-\left(2 \frac{\Delta x}{\left(1-\eta_{1}\right) \Delta t} u_{o} \jmath \sin (\vartheta)-2 u_{o}^{2} \sin ^{2}(\vartheta)\right)}{\left(\frac{\Delta x}{\left(1-\eta_{1}\right) \Delta t}+u_{o}(\jmath \sin (\vartheta)+1-\exp (-\jmath \vartheta))\right)} \\
\cdot\left(\frac{2}{u_{o}+\Delta x / \Delta t}(\cos (\vartheta)-1)\right)-\sin ^{2}(\vartheta)
\end{gathered}
$$

Because the velocity and acceleration at time level $n+\delta$ (resp. $n+1)$ are only calculated when the Gauss-Seidel coupling iterations within the first (resp. second) sub-step have converged, Eq. (9b) and Eq. (9c) (resp. Eq. (12b) and Eq. (12c)) are of no importance for the stability of the coupling iterations.

\subsection{Discussion}

It can be observed that the error amplification factor $\mu$ for the time discretization with the backward Euler and Newmark scheme (Eq. (18)) is almost the same as for the first (Eq. (21)) and second (Eq. (23)) sub-step in the composite time 
discretization. The error amplification factor is consequently determined by the following expressions.

$$
\mu_{1}=\frac{\frac{\rho_{f}}{\rho_{s}}}{\frac{\rho_{f} r_{o}^{2}\left(1-\nu^{2}\right)}{\sigma_{1} E \Delta t^{2}}+\frac{4 A r_{o}^{2}\left(1-\nu^{2}\right)}{E h \Delta x^{4}} \frac{\rho_{f}}{\rho_{s}}(\cos (\vartheta)-1)^{2}}
$$

and

$$
\begin{gathered}
\left(\frac{\Delta x}{\sigma_{2} \Delta t}+u_{o}(\jmath \sin (\vartheta)+1-\exp (-\jmath \vartheta))\right) \\
\cdot\left(2 \frac{\Delta x}{\sigma_{2} \Delta t}+2 u_{o} \jmath \sin (\vartheta)\right) \\
\mu_{2}=\frac{\rho_{f} r_{o}\left(1-\nu^{2}\right)}{E h} \cdot \frac{-\left(2 \frac{\Delta x}{\sigma_{2} \Delta t} u_{o} \jmath \sin (\vartheta)-2 u_{o}^{2} \sin ^{2}(\vartheta)\right)}{\left(\frac{\Delta x}{\sigma_{2} \Delta t}+u_{o}(\jmath \sin (\vartheta)+1-\exp (-\jmath \vartheta))\right)} \\
\cdot\left(\frac{2}{u_{o}+\Delta x / \Delta t}(\cos (\vartheta)-1)\right)-\sin ^{2}(\vartheta)
\end{gathered}
$$

The parameters $\sigma$ in the equation above are

$$
\begin{aligned}
& \sigma_{1}=\beta \\
& \sigma_{2}=1
\end{aligned}
$$

for the backward Euler and Newmark time discretization. In the first sub-step of the composite time discretization, these parameters are

$$
\begin{aligned}
\sigma_{1} & =\left(\frac{\delta}{2}\right)^{2} \\
\sigma_{2} & =\frac{\delta}{2}
\end{aligned}
$$

and in the second sub-step they are

$$
\begin{aligned}
\sigma_{1} & =\left(\frac{1-\delta}{2-\delta}\right)^{2} \\
\sigma_{2} & =\frac{1-\delta}{2-\delta}
\end{aligned}
$$


Because $(\cos (\vartheta)-1)^{2}$ is always positive and $(\cos (\vartheta)-1)$ always negative, the second and third term in the denominator of $\mu_{1}$ always reduce the error amplification. The error amplification will thus always be mitigated by increasing the parameters $A$ and $B$ which account for the interaction between segments of the tube. Taking the interaction between the segments into account in the structural model should thus facilitate the convergence of the coupling iterations compared to a simulation with an independent-rings model. Both $A$ and $B$ appear only once in the expression for $\mu$ which makes it easy to understand their effect and consequently they can be set to zero in the remainder of the analysis. With $A=B=0$, $\mu_{1}$ is the same for all wave numbers.

Increasing the Newmark parameter $\beta$ increases the error amplification but the change is only significant if $\frac{\rho_{f}}{\rho_{s}} \ll \frac{\rho_{f} r_{o}^{2}\left(1-\nu^{2}\right)}{E \beta \Delta t^{2}}$. The parameter $\gamma$ of the Newmark scheme has no effect on the stability of the coupling iterations. The parameter $\delta$ of the composite time integration has a more complex influence on $\mu$. However, the parameters of the time integration are usually not varied over a wide range so their effect is of minor importance.

The effect of the time step on the stability is frequently important and it is more complex. The factor $\mu_{1}$ is proportional to $\Delta t^{2}$ if $\frac{\rho_{f}}{\rho_{s}} \ll \frac{\rho_{f} r_{o}^{2}\left(1-\nu^{2}\right)}{E \sigma_{1}}$ and if the relative contribution of the terms due to the interaction between the segments of the structure is small. If $u_{o} \ll \frac{\Delta x}{\Delta t}$ then $\mu_{2}$ becomes proportional to $\frac{1}{\Delta t^{2}}$; otherwise $\Delta t$ only influences $\mu_{2}$ for the lowest and highest wave numbers $(\vartheta \approx 0$ or $\pi)$. Consequently, there are three situations for the effect of the time step on the stability of the coupling iterations for this particular model, with $v_{o}=\sqrt{\frac{E \sigma_{1}}{\rho_{s}\left(1-\nu^{2}\right)}}$ :

- $\mu \sim \Delta t^{2}$ : if $\Delta t \ll \frac{r_{o}}{v_{o}}$ and $\Delta t \gg \frac{\Delta x}{u_{o}}$;

- $\mu \approx$ constant: if both $\Delta t \ll \frac{r_{o}}{v_{o}}$ and $\Delta t \ll \frac{\Delta x}{u_{o}}$ or if both $\Delta t \gg \frac{r_{o}}{v_{o}}$ and $\Delta t \gg \frac{\Delta x}{u_{o}}$

- $\mu \sim \frac{1}{\Delta t^{2}}$ : if $\Delta t \gg \frac{r_{o}}{v_{o}}$ and $\Delta t \ll \frac{\Delta x}{u_{o}}$.

If $\Delta t$ is varied over a wide range, the effect of $\Delta t$ on $\mu$ might change throughout that variation as $\Delta t$ determines which of the above situations is appropriate. The time step $\Delta t$ will have no significant influence on the error amplification factor $\mu$ if the former is sufficiently far outside the range

$$
\left[\min \left(\frac{r_{o}}{v_{o}}, \frac{\Delta x}{u_{o}}\right), \max \left(\frac{r_{o}}{v_{o}}, \frac{\Delta x}{u_{o}}\right)\right] .
$$


If $\frac{r_{o}}{v_{o}} \ll \frac{\Delta x}{u_{o}}$ then $\mu$ is proportional to $\frac{1}{\Delta t^{2}}$ when $\Delta t$ lies in the range given above. On the contrary, if $\frac{r}{v_{o}} \gg \frac{\Delta x}{u_{o}}$ then $\mu$ is proportional to $\Delta t^{2}$ for $\Delta t$ in that range.

Fig. 2 depicts the evolution of $\mu$ as function of $\Delta t$ with parameters that approximate the flow in an artery (see Tab. 1). One can see that $\mu$ is proportional to $\frac{1}{\Delta t^{2}}$ for $\Delta t \in\left[5 \times 10^{-4}, 5 \times 10^{-3}\right]$ and constant for $\Delta t$ outside that range. Fig. 4 shows $\mu$ as a function of the wave number for four different time steps with again the increase of $\mu$ for decreasing $\Delta t$ as long as $\Delta t \in\left[5 \times 10^{-4}, 5 \times 10^{-3}\right]$ and no further significant change outside that range. These figures have been created with the parameter values listed in Tab. 1 which approximate the flow in a large artery for backward Euler time discretization of the flow equations and Newmark time discretization of the structural equations. Fig. 3 and Fig. 5 depict the error amplification in the first and second sub-step of the composite time discretization and they are nearly identical to the figures for the backward Euler and Newmark time discretization. The range $\left[5 \times 10^{-4}, 5 \times 10^{-3}\right]$ is indicated with vertical dotted lines in Fig. 2 and Fig. 3. The stability limit $(\mu=1)$ is marked with a horizontal dotted line in Fig. 2, Fig. 3, Fig. 4 and Fig. 5.

The error amplification factor for the low wave numbers still depends on the time step's size when $\Delta t \in\left[5 \times 10^{-3}, 10^{-1}\right]$ in Fig. 2. For the highest wave number $(\vartheta=\pi)$, the influence of the time step's size remains present when $\Delta t$ goes to infinity. As mentioned above, $\mu_{2}$ is still influenced by $\Delta t$ for the lowest and highest wave numbers $\left(\vartheta \approx 0\right.$ or $\pi$ ) when $\Delta t>\frac{\Delta x}{u_{o}}$.

The foregoing explains the conclusion in [9] that $\mu \sim \frac{1}{\Delta t^{2}}$ if the inertia of the structure is neglected $\left(\rho_{s}=0\right)$ and $\Delta t \ll \frac{x}{u_{o}}$. The latter inequality is also the limit for which the discretization of the flow equations can be used and consequently no larger time steps were used in our previous study [9] such that the relation $\mu \sim \frac{1}{\Delta t^{2}}$ was always satisfied.

\subsection{Limitations}

The Fourier error analysis employs linearized equations and it does not take into account the boundary conditions. The result of the Fourier analysis also depends on the numerical schemes that are used for the discretization in space of the flow equations and structural equations.

\section{Nonlinear numerical experiments}

Nonlinear simulations of the flow in a flexible tube are used to verify the conclusions of the linear analysis, especially with regard to the effect of the time step as this effect is the most important one. All simulations have been performed with 
the backward Euler time discretization for the flow equations and the Newmark scheme for the structural equations. The difference in accuracy of the result with respect to the composite time discretization and the stability of a sequence of time steps fall outside the scope of this work.

A fluid velocity of $u_{o}+\frac{u_{o}}{100} \sin (2 \pi t)$ has been applied at the inlet of the tube and zero pressure is imposed at the outlet of the tube. The structure is initially at rest and both $A$ and $B$ have been set to zero. The tube is discretized in 100 segments with the same length. The values from Tab. 1 have been used again for the geometry and for the properties of the materials. For these values, the error analysis predicts that the error amplification factor $\mu$ will increase for a decreasing time step in the range $\left[5 \times 10^{-4}, 5 \times 10^{-3}\right]$ and that $\mu$ will be constant outside this range (see Fig. 2).

Simulations with 100 time steps have been performed for different values of $\Delta t$ and with different coupling algorithms. The number of coupling iterations per time step (averaged over the 100 time steps in the simulation) is a measure for the error amplification factor $\mu$. A high error amplification factor means that the number of coupling iterations will be high and the Gauss-Seidel iterations will diverge if $\mu>1$ for some wave numbers. The $L_{2}$-norm of the residual is reduced with 3 orders of magnitude with respect to its initial value in the time step. In Fig. 6, the average number of coupling iterations per time step is depicted for different sizes of the time step and the range $\left[5 \times 10^{-4}, 5 \times 10^{-3}\right]$ is again indicated with vertical dotted lines.

Gauss-Seidel (GS) iterations converge quickly for a large time step $\left(10^{-1} \mathrm{~s}\right)$ because the error amplification is smaller than one for all wave numbers in this region as shown in Fig. 2. When the time step decreases, the convergence of the Gauss-Seidel iterations becomes slow, e.g. on average 28 Gauss-Seidel coupling iterations per time step were required for a time step of $10^{-2} \mathrm{~s}$. The Gauss-Seidel iterations diverged in the first time step when $\Delta t$ was less than $10^{-2} \mathrm{~s}$.

It is thus impossible to verify the conclusions of the stability analysis over a wide range of time steps by performing simulations with the Gauss-Seidel coupling algorithm because the error amplification factor for the low wave numbers would be larger than one in the simulations with a small time step which would cause divergence of the Gauss-Seidel coupling iterations. Other coupling algorithms, such as IQN-ILS, have to be used for a partitioned simulation with small time steps. The IQN-ILS algorithm uses information from the previous coupling iterations in the current time step to approximate the inverse of the Jacobian of the coupled problem written as a root-finding equation. The IQN-ILS algorithm constructs a vector space that grows during the coupling iterations and it behaves 
like a Newton algorithm for the part of the error in this vector space and like Gauss-Seidel iterations for the other part. More information on this technique can be found in [5].

Fig. 6 shows that the number of IQN-ILS coupling iterations per time step is almost the same for $\Delta t=3 \cdot 10^{-1} \mathrm{~s}$ to $3 \cdot 10^{-2} \mathrm{~s}$. Between $\Delta t=10^{-2} \mathrm{~s}$ and $3 \cdot 10^{-4} \mathrm{~s}$, the number of IQN-ILS iterations increases steadily with decreasing time step. The number of coupling iterations per time step is almost the same for $\Delta t=10^{-4} \mathrm{~s}$ to $10^{-5} \mathrm{~s}$. Consequently, the number of coupling iterations per time step (Fig. 6) and the error amplification factor (Fig. 2) have the same behavior.

The increase of the number of IQN-ILS coupling iterations with a decreasing time step can be mitigated by using information from the coupling iterations in the previous time steps instead of only information from the current time step. Fig. 6 illustrates that the number of coupling iterations per time step is reduced significantly if the information from the four previous time steps, denoted as IQN$\operatorname{ILS}(4)$, is also used.

\section{Conclusions}

Fourier error analysis on Gauss-Seidel iterations between the flow solver and structural solver for the flow in a flexible tube has been performed. The error amplification factor is reduced compared to the independent-rings model by including the interaction between the segments of the tube in the structural model. For parameter values that approximate the flow in a large artery, the error amplification factor of Gauss-Seidel coupling iterations increases if the size of the time step decreases within a certain range of the time step's size. Outside that range, the time step has little effect on the error amplification factor. Backward Euler time discretization for the flow equations combined with Newmark time discretization of the structural equations has been compared with composite time discretization of both the flow and the structural equations and it has been shown that the time discretization has almost no effect on the stability of the coupling iterations within the time step. The stability analysis of the Gauss-Seidel iterations is confirmed by the variation of the number of IQN-ILS coupling iterations as a function of the size of the time step in nonlinear simulations.

\section{Acknowledgments}

J. Degroote gratefully acknowledges a Ph.D. fellowship of the Research Foundation - Flanders (FWO). 


\section{References}

[1] U. Küttler, W. Wall, Fixed-point fluid-structure interaction solvers with dynamic relaxation, Computational Mechanics 43 (1) (2008) 61-72, DOI: 10.1007/s00466-008-0255-5.

[2] M. Fernandez, M. Moubachir, A Newton method using exact Jacobians for solving fluid-structure coupling, Computers \& Structures 83 (2-3) (2005) 127-142.

[3] S. Michler, Efficient numerical methods for fluid-structure interaction, Phd thesis, TUDelft, Department of Aerospace Design, Integration \& Operations, 2005.

[4] J. Vierendeels, L. Lanoye, J. Degroote, P. Verdonck, Implicit coupling of partitioned fluid-structure interaction problems with reduced order models, Computers \& Structures 85 (11-14) (2007) 970-976.

[5] J. Degroote, K.-J. Bathe, J. Vierendeels, Performance of a new partitioned procedure versus a monolithic procedure in fluid-structure interaction, Computers \& Structures 87 (11-12) (2009) 793-801.

[6] M. Lesoinne, C. Farhat, A higher-order subiteration free staggered algorithm for non-linear transient aeroelastic problems, AIAA Journal 36 (9) (1998) $1754-1756$.

[7] C. Förster, W. Wall, E. Ramm, Artificial added mass instabilities in sequential staggered coupling of nonlinear structures and incompressible viscous flows, Computer Methods in Applied Mechanics and Engineering 196 (7) (2007) 1278-1293.

[8] P. Causin, J.-F. Gerbeau, F. Nobile, Added-mass effect in the design of partitioned algorithms for fluid-structure problems, Computer Methods in Applied Mechanics and Engineering 194 (42-44) (2005) 4506-4527.

[9] J. Degroote, P. Bruggeman, R. Haelterman, J. Vierendeels, Stability of a coupling technique for partitioned solvers in FSI applications, Computers \& Structures 86 (23-24) (2008) 2224-2234.

[10] A. Quarteroni, M. Tuveri, A. Veneziani, Computational vascular fluid dynamics: problems, models and methods, Computing and Visualization in Science 2 (2000) 163-197. 
[11] N. Newmark, A Method of Computation for Structural Dynamics, ASCE Journal of the Engineering Mechanics Division 85 (3) (1959) 67-94.

[12] R. Bank, W. Coughran, W. Fichter, E. Grosse, D. Rose, R. Smith, Transient simulations of silicon devices and circuits, IEEE Transactions on ComputerAided Design of Integrated Circuits and Systems 4 (4) (1985) 436-451.

[13] K.-J. Bathe, M. Baig, On a composite implicit time integration procedure for nonlinear dynamics, Computers \& Structures 83 (31-32) (2005) 2513-2524.

[14] K.-J. Bathe, Conserving Energy and Momentum in Nonlinear Dynamics: A Simple Implicit Time Integration Scheme, Computers \& Structures 85 (7-8) (2007) 437-445.

[15] J. Vierendeels, K. Riemslagh, E. Dick, A multigrid semi-implicit linemethod for viscous incompressible and low-Mach-number flows on high aspect ratio grids, Journal of Computational Physics 154 (2) (1999) 310-341.

[16] K.-J. Bathe, The inf-sup condition and its evaluation for mixed finite element methods, Computers \& Structures 79 (2) (2001) 243-252,971. 


\section{Figures and tables}

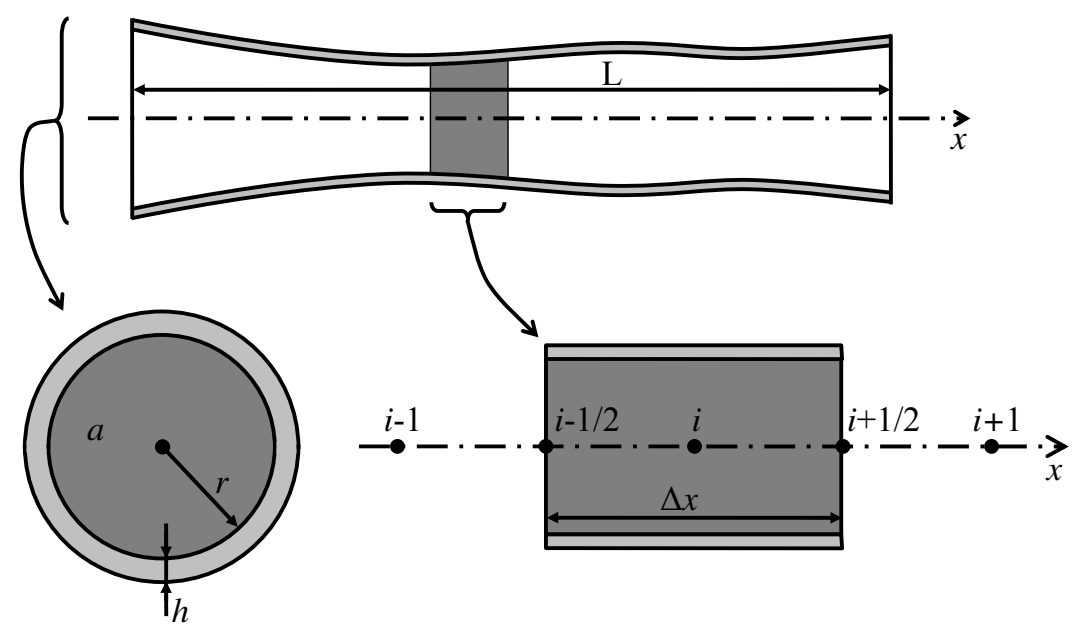

Figure 1: The geometry of the flexible tube with details of the cross section $a$ and a control volume used in the discretization of the flow equations. 


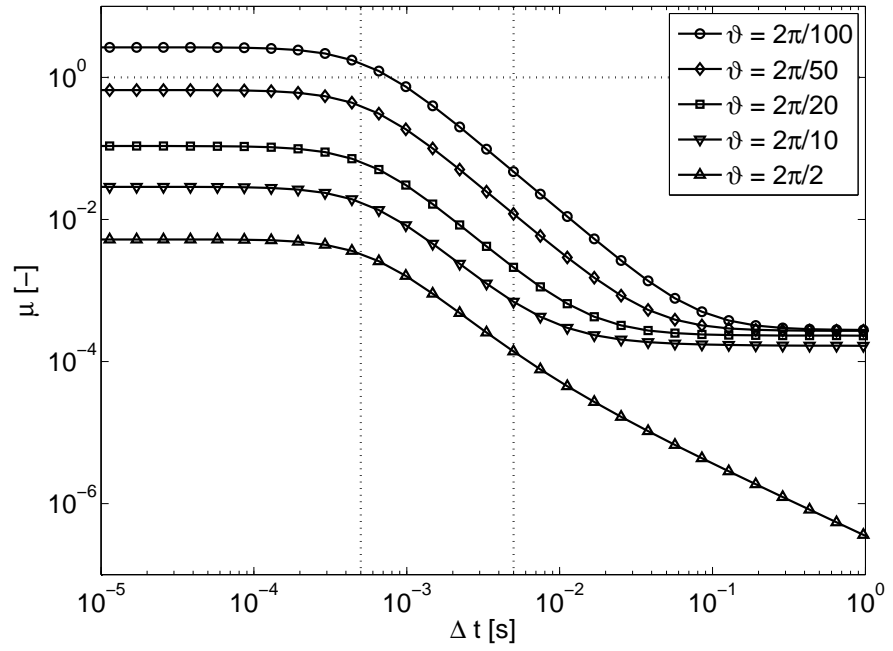

Figure 2: The error amplification factor $\mu$ as a function of the size of the time step $\Delta t$ if backward Euler and Newmark time discretization is used. 


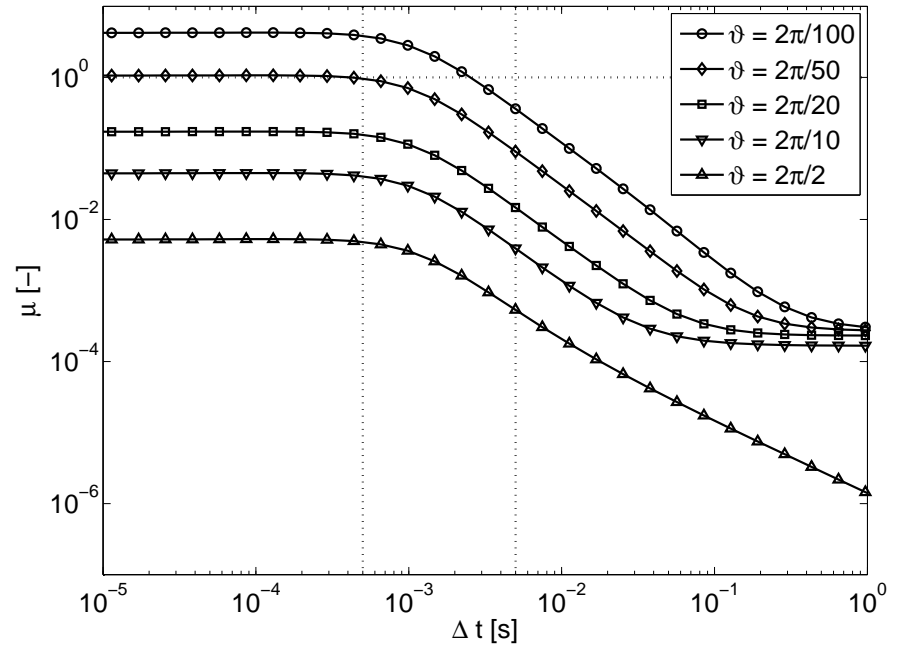

(a)

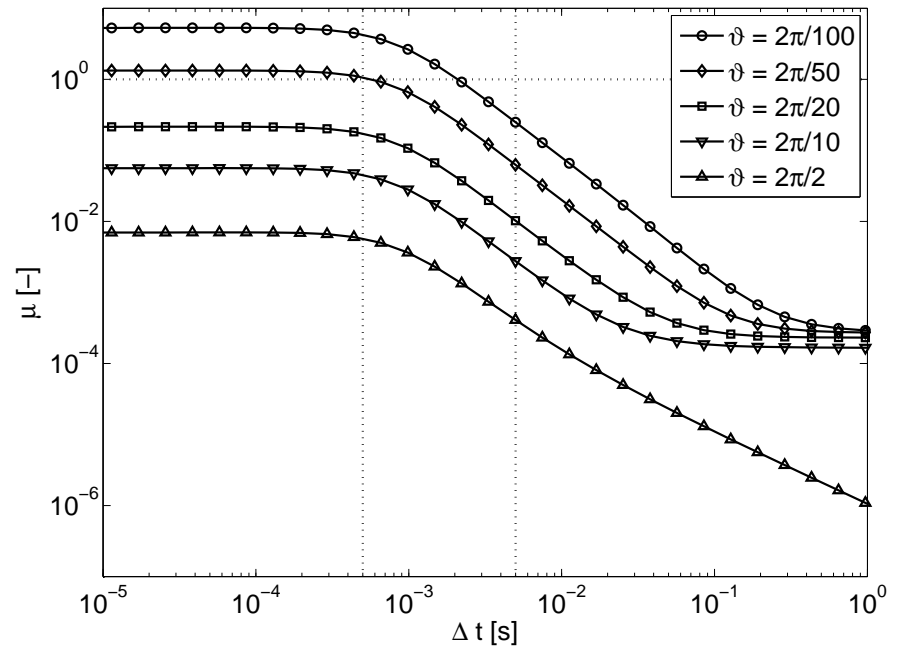

(b)

Figure 3: The error amplification factor $\mu$ as a function of the size of the time step $\Delta t$ if composite time discretization is used. (a) First sub-step and (b) second sub-step. 


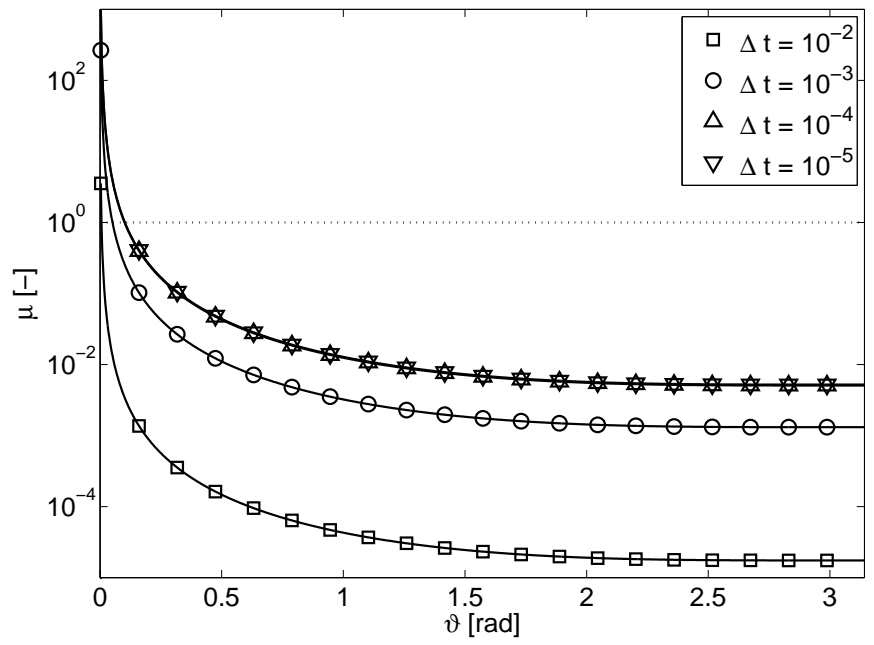

Figure 4: The error amplification factor $\mu$ as a function of the wave number $\vartheta$ if backward Euler and Newmark time discretization is used. Low wave numbers have a higher error amplification factor than high wave numbers. 


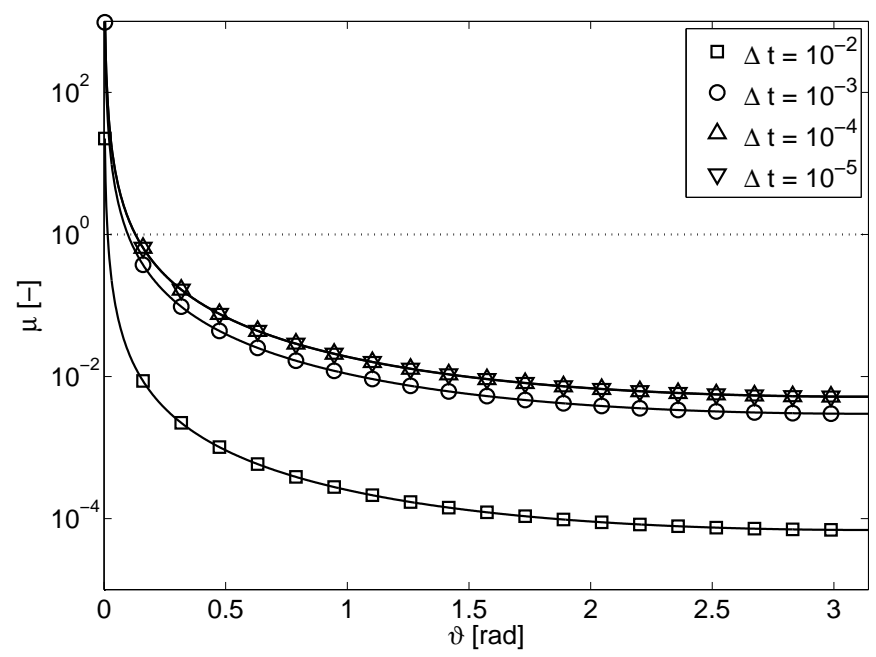

(a)

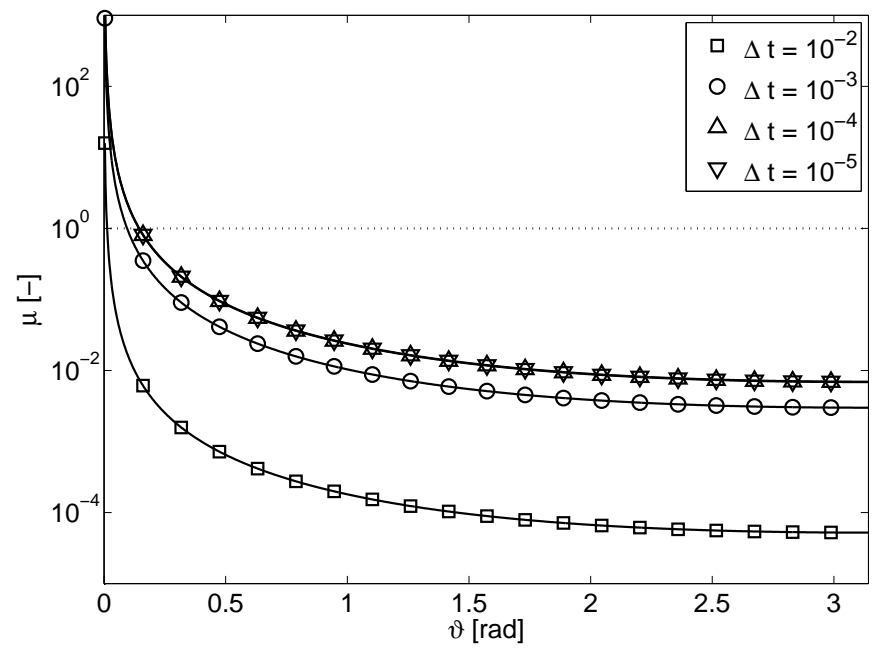

(b)

Figure 5: The error amplification factor $\mu$ as a function of the wave number $\vartheta$ if composite time discretization is used. (a) First sub-step and (b) second sub-step. 


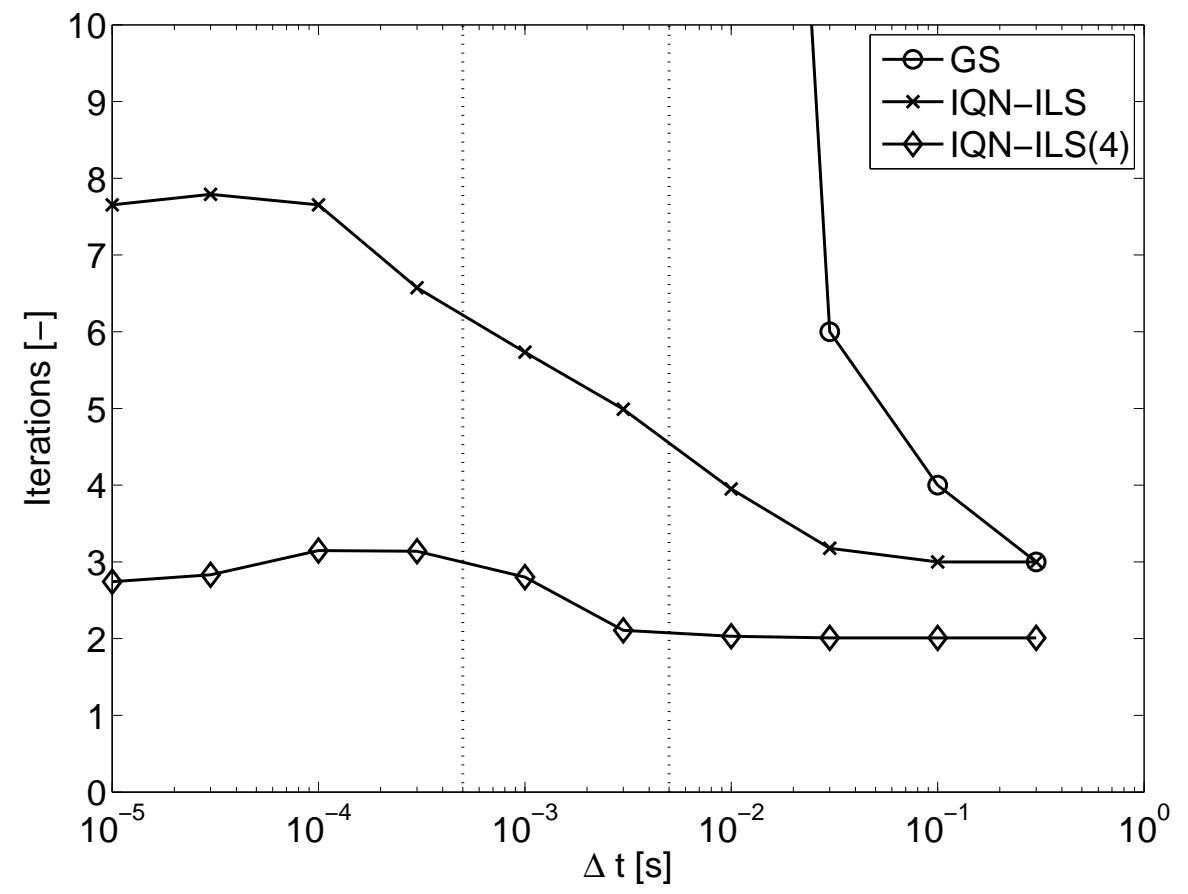

Figure 6: The number of coupling iterations per time step (averaged over 100 time steps) for different sizes of the time step. The Gauss-Seidel (GS) iterations only converge for a large time step whereas the iterations of the interface quasi-Newton algorithm with approximate inverse Jacobian from a least-squares model (IQN-ILS) converge for much smaller time steps. 


\begin{tabular}{|c|rl|}
\hline$E$ & 300000 & $\mathrm{~N} / \mathrm{m}^{2}$ \\
$\nu$ & 0.4 & \\
$L$ & 0.05 & $\mathrm{~m}$ \\
$h$ & 0.001 & $\mathrm{~m}$ \\
$r_{o}$ & 0.005 & $\mathrm{~m}$ \\
$u_{o}$ & 0.1 & $\mathrm{~m} / \mathrm{s}$ \\
$\rho_{f}$ & 1000 & $\mathrm{~kg} / \mathrm{m}^{3}$ \\
$\rho_{s}$ & 1200 & $\mathrm{~kg} / \mathrm{m}^{3}$ \\
$\beta$ & $1 / 4$ & \\
$\gamma$ & $1 / 2$ & \\
$\delta$ & $1 / 2$ & \\
\hline
\end{tabular}

Table 1: Parameters that are used as approximate values for the flow in an artery. 